\title{
The Numerical Solution of Fractional Diffusion Equation by Using Local Polynomial Regression
}

\author{
H. CAGLAR ${ }^{a, *}$, N. CAGLAR ${ }^{b}$, M. OzER $^{c}$ \\ ${ }^{a}$ Istanbul Kultur University, Department of Mathematics-Computer, Istanbul, Turkey \\ ${ }^{b}$ Istanbul Kultur University, Faculty of Economic and Administrative Science, Istanbul, Turkey \\ ${ }^{c}$ Istanbul Kultur University, Department of Physics, Istanbul, Turkey
}

\begin{abstract}
In this paper, we apply the local polynomial regression for the solution of the one-dimensional fractional diffusion equation. The Caputo fractional derivative is used for the formulation. A numerical example is given to show the application of the present method. The effect of the fractional order $(\alpha)$ of the equation is illustrated by changing $\alpha$ from 1.01 to 1.99 .
\end{abstract}

DOI: $10.12693 /$ APhysPolA.125.551

PACS: 02.60.-x, 02.60.Lj

\section{Introduction}

Diffusion equations are an important part of partial differential equations because of their potential applications in science, engineering, and social sciences. On the other hand, the fractional derivatives have been started to apply in several systems in the past few decades. Recently an extensive work has appeared in literature dealing with one- and two-dimensional partial diffusion equations [1-6].

It is well known that the one-dimensional fractional diffusion equation is defined as:

$$
\frac{\partial u(x, t)}{\partial t}=d(x) \frac{\partial^{\alpha} u(x, t)}{\partial x^{\alpha}}+q(x, t) .
$$

To Eq. (1) we attach the initial conditions and boundary condition

$$
\begin{aligned}
& u(x, 0)=f(x), \quad 0 \leq x \leq 1, \\
& u(0, t)=g_{0}(t), \quad t \geq 0, \\
& u(1, t)=g_{1}(t), \quad t \geq 0,
\end{aligned}
$$

on a finite domain $0 \leq x \leq 1$, for $t \geq 0$. Here, $d(x)$ represents the diffusion coefficient and $q(x, t)$ - the source/sink function. Sources provide energy or material to the system where sinks absorb energy or material. Equation (1) becomes the classical diffusion equation for $\alpha=2$. It models a superdiffuse flow for $1<\alpha<2$ and a classical advective flow for $\alpha=1$ [2].

In this paper, the local polynomial regression (LPR) is applied for the numerical solution of one-dimensional fractional diffusion equation. The paper has been organized as follows. Necessarily theoretical background is given in Sect. 2. In Sect. 3, LPR solutions for partial diffusion equation are applied. A numerical example is presented in Sect. 4.

*corresponding author; e-mail: s.caglar@iku.edu.tr

\section{Theoretical background}

In this section, we recall the Caputo fractional derivative and the local polynomial regression.

\subsection{Caputo fractional derivative}

There are various kinds of fractional derivatives that one can use for the fractional calculations. The widely used fractional derivatives are the Grunwald-Letnikov, the Riemann-Liouville, and the Caputo fractional derivatives. The Caputo fractional derivative is a regularization in the time origin for the Riemann-Liouville fractional derivative $[7,8]$. A nice comparison of these definitions from the view point of their applications in physics and engineering can be found in $[9,10]$.

In this paper, we use the Caputo fractional derivative that is defined as follows [11]:

$$
\begin{gathered}
D_{* x}^{\alpha}=J^{m-\alpha} D^{m} f(x)=\frac{1}{\Gamma(m-\alpha)} \\
\quad \times \int_{0}^{x}(x-t)^{m-\alpha-1} f(m)(t) \mathrm{d} t
\end{gathered}
$$

for $m-1<\alpha \leq m$ and $m \in N$. The Caputo fractional derivative is considered here because the fractional diffusion equation is transformed to the integro-differential equation by using it.

\subsection{Local polynomial regression}

Suppose that the $(p+1)$-th derivative of $x(t)$ at point $t_{0}$ exists. We approximate the unknown regression function $y(t)$ locally at $t_{0}$ by a polynomial of order $p$. The theoretical justification is that we can approximate, in a neighborhood of $t_{0}, y(t)$ using a Taylor expansion

$$
y(t) \approx \sum_{k=0}^{p} \beta_{k}\left(t_{i}-t_{0}\right)^{k},
$$

where

$$
\beta_{k}=\frac{t(k)\left(t_{0}\right)}{k !} .
$$

This polynomial, used to approximate the unknown function locally at $t_{0}$, is obtained by solving a locally weighted least squares regression problem, i.e. by minimizing 


$$
\sum_{i=1}^{n}\left[Y_{i}-\sum_{k=0}^{p} \beta_{k}\left(t_{i}-t_{0}\right)^{k}\right]^{2} K \frac{\left(t_{i}-t_{0}\right)}{h},
$$

where $h$ is the smoothing parameter and $K$ is a kernel function. The local polynomial kernel regression estimate $\beta$ is given by $\beta=\left(X^{\mathrm{T}} W X\right)^{-1} X^{\mathrm{T}} W Y$, where $x=\left(\left(t_{i}-\right.\right.$ $\left.\left.t_{0}\right)^{i-1}\right)_{1 \leq i \leq n}, 1 \leq j \leq p+1, W=\operatorname{diag}\left[K_{h}\left(t_{i}-t_{0}\right)\right]$ the vectors $\bar{Y}=\left(Y_{1}, Y_{2}, \ldots, Y_{n}\right)^{\prime}$ and $\beta=\left(\beta_{0}, \beta_{1}, \ldots, \beta_{p}\right)^{\prime}$. A detailed description can be found in [12].

3. LPR solutions for partial diffusion equation

Difference schemes for this first problem are considered as following:

$$
\frac{u_{i+1}-u_{i}}{\Delta t}=d(x) \frac{\partial^{\alpha} u(x, t)}{\partial x^{\alpha}}+q(x, t),
$$

where $\Delta t=k$ :

$$
-k d(x) u_{i+1}(\alpha)+u_{i+1}=u_{i}+k q(x, t)
$$

and the initial conditions are given in (2),

$$
u(x, 0)=f(x)=u_{0} .
$$

Substituting (11) in (10) then is obtained as follows:

$$
\begin{aligned}
& t=0+k, \quad-k d(x) u_{1}^{(\alpha)}+u_{1}=u_{0}+k q(x k), \\
& t=0+2 k, \quad-k d(x) u_{2}^{(\alpha)}+u_{2}=u_{i}+k q(x, 2 k), \\
& \vdots \quad \vdots \\
& t=0+n k, \quad-k d(x) u_{n}^{(\alpha)}+u_{n}=u_{n-1}+k q(x, n k) .
\end{aligned}
$$

In this section, the LPR method for solving Eq. (1) is outlined. Let Eq. (6) be an approximate solution of Eq. (1):

$$
y(x)=\sum_{j=0}^{p} \beta_{j}\left(x_{i}-x_{0}\right)^{j},
$$

where $x_{1}=\alpha, x_{2}, \ldots x_{n}=b$ and it is required that the approximate solution (15) satisfies the boundary value problems at the points $x=x_{i}$. Putting (15) in (12), it follows that:

$$
\begin{aligned}
& -k d\left(x_{i}\right) \sum_{j=m+1}^{p} \frac{1}{\gamma(m-\alpha)} \int_{0}^{x_{i}}\left(x_{i}-s\right)^{m-\alpha-1} \\
& \times \beta_{j} j(j-1) \ldots(j-m)\left(s-x_{0}\right)^{j-m-1} \mathrm{~d} s \\
& +\sum_{j=0}^{p} \beta_{j}\left(x-x_{0}\right)^{j}=u_{0}+k q(x, k), \\
& \quad a \leq t \leq b, \quad a \leq s \leq b .
\end{aligned}
$$

This leads to the system

$$
\begin{aligned}
& i=1, \quad a_{i, j}=\beta_{j}\left(x_{1}-x_{0}\right)^{j}, \quad j=0, p, \quad y(i)=g_{0}(k) \\
& i=2, \quad n-1, \quad b_{i j}=\beta_{j}\left(x_{i}-x_{0}\right)^{j}, \quad j=0, p, \\
& c_{i j}=\frac{1}{\gamma(m-\alpha)} \int_{0}^{t_{i}}\left(t_{i}-s\right)^{m-\alpha-1} \beta_{j} j(j-1) \ldots \\
& \ldots(j-m)\left(s-t_{0}\right)^{j-m-1} \mathrm{~d} s, \quad j=m+1, p, \\
& y(i)=u_{0}(i)+k q\left(x_{i} k\right) \text {, } \\
& i=n, \quad a_{n, j}=\beta_{j}\left(x_{n}-x_{0}\right)^{j}, \quad j=0, m, \\
& y(i)=g_{1}(k) \text {. }
\end{aligned}
$$

Then, the matrix form can be written as follows by using Eqs. (17)-(19).

$$
X=
$$$$
\left[\begin{array}{cccccc}
a_{1,0} & a_{1,1} & \ldots & \ldots & \ldots & a_{1, p} \\
b_{2,0} & b_{2,1} & \ldots & b_{2, m+1}+c_{2, m+1} & \ldots & b_{2, p}+c_{2, p} \\
b_{3,0} & b_{3,1} & \ldots & b_{3, m+1}+c_{3, m+1} & \ldots & b_{3, p}+c_{3, p} \\
\vdots & \vdots & \vdots & \vdots & \vdots & \vdots \\
b_{n-1,0} & b_{n-1,1} & \ldots & b_{n-1, m+1}+c_{n-1, m+1} & \ldots & b_{n-1, p}+c_{n-1, p} \\
a_{n, 0} & a_{n, 1} & \ldots & \ldots & \ldots & a_{n, m}
\end{array}\right]
$$

$$
Y=\left[\begin{array}{l}
y(1) \\
\vdots \\
y(n)
\end{array}\right] .
$$

Putting (20) in $=\left(X^{\mathrm{T}} W X\right)^{-1} X^{\mathrm{T}} W Y$, then estimated set of coefficients $\beta_{i}$ are obtained by solving matrix system. Therefore, approximate solution (15) is obtained.

\section{A numerical example}

In this section, we consider the following one-dimensional fractional differential equation, as taken in the literature $[2,5,6]$ :

$$
\frac{\partial u(x, t)}{\partial t}=d(x) \frac{\partial^{1.8} u(x, t)}{\partial x^{1.8}}+q(x, t),
$$

on a finite domain $0<x<1$, with the diffusion coefficient

$$
d(x)=\Gamma(2.2) x^{2.8} / 6,
$$

the source/sink function

$$
q(x, t)=-(1+x) \mathrm{e}^{-t} x^{3},
$$

subject to the initial condition,

$$
u(x, 0)=x^{3}, \quad \text { for } 0<x<1,
$$

and the boundary conditions

$$
u(0, t)=0, \quad u(1, t)=\mathrm{e}^{-t}, \quad \text { for } t>0 .
$$

Note that the exact solution for $\alpha=2$ is

$$
y(x)=\mathrm{e}^{-t} x^{3} .
$$

\section{TABLE}

The maximum absolute errors for $m=5$ (polynomial degree) and $k=0.01$ (time step).

\begin{tabular}{c|c|c}
\hline \hline$\alpha$ & $n=11$ & $n=21$ \\
\hline 1.01 & 0.2068903 & 0.2229955 \\
1.20 & 0.1952389 & 0.2086801 \\
1.40 & 0.1760765 & 0.1855386 \\
1.60 & 0.1471001 & 0.1525271 \\
1.80 & 0.1044667 & 0.1051868 \\
1.99 & 0.0491571 & 0.0475289
\end{tabular}

Table shows the maximum absolute errors between for the known exact solution $(\alpha=2)$ and for the following fraction orders: $1.01,1.20,1.40,1.60,1.80,1.99$. The behaviour of the solution for different values of $\alpha$ is very 
instructive for the aspect of modelling. It is clearly seen that the magnitude of the errors decreases by increasing the fraction order from 1 to 2 . For the fractional diffusion equation satisfactory results is received, which are illustrated by some numerical experiments (see Fig. 1). All computations are carried out using MATLAB 6.5.

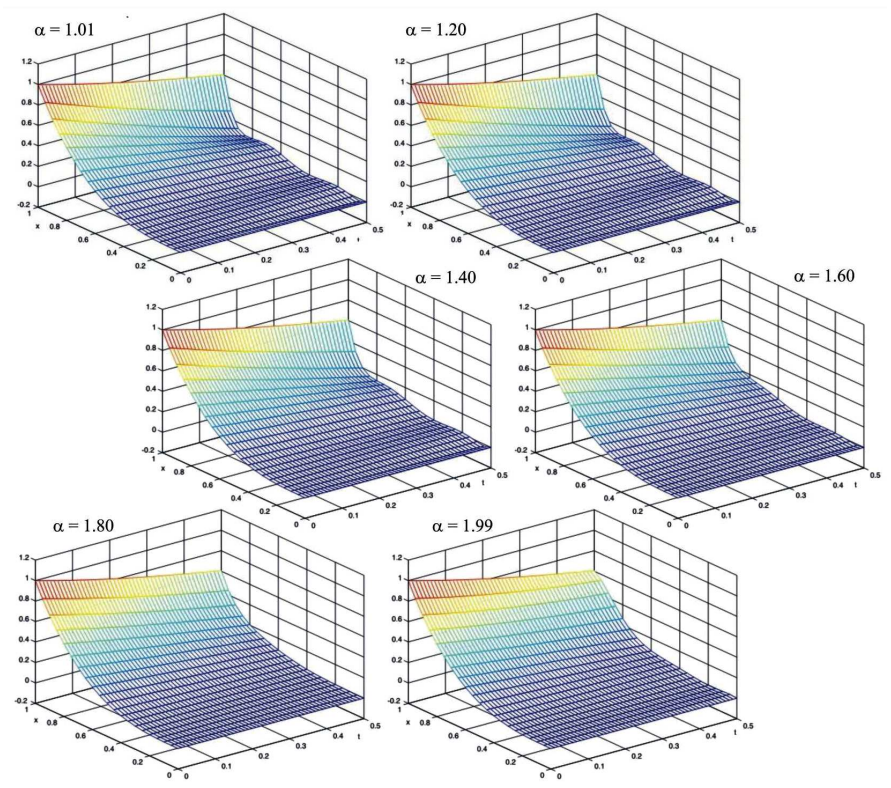

Fig. 1. Results for different values of $\alpha$.

\section{Conclusions}

In this paper, the LPR is applied for the numerical solution of one-dimensional fractional diffusion equation.
The fractional derivative is defined in the Caputo sense. The method is applied on a test problem where the $\alpha$ is equal to $1.01,1.20,1.40,1.60,1.80$, and 1.99 . It may be concluded that the LPR is very powerful and efficient method in finding solutions for these kind of problems.

\section{References}

[1] M.M. Meerschaert, H.P. Scheffer, C. Tadjeran, J. Comput. Phys. 211, 249261 (2006).

[2] C. Tadjeran, M.M. Meerschaert, H.P. Scheffer, J. Comput. Phys. 213, 205213 (2006).

[3] M.M. Meerschaert, C. Tadjeran, Appl. Num. Math. 56, 80 (2006).

[4] C. Tadjeran, M.M. Meerschaert, J. Comput. Phys 220, 813 (2007).

[5] S.S. Ray, K.S. Chaudhuri, R.K. Bera, Appl. Math. Comput. 196, 294302 (2008).

[6] S.S. Ray, Commun. Nonlinear Sci. Numer. Simul. 13, 1311 (2008).

[7] M. Caputo, Geophys. J. Roy. Astron. Soc. 13, 529539 (1967).

[8] R. Goreno, F. Mainardi, Arch. Mech. 50, 377388 (1998).

[9] I. Podlubny, L. Dorcak, I. Kostial, Decision Control 5, 4985 (1997).

[10] J. Sabatier, O. Prakash Agrawal, J.A. Tenreiro Machado, Advances in Fractional Calculus: Theoretical Developments and Applications in Physics and Engineering, Springer, Netherlands 2007.

[11] C.P. Li, W.H. Deng, Appl. Math. Comput. 187, 777 (2007).

[12] H. Caglar, N. Caglar, Appl. Math. Comput. 186, 952 (2007). 